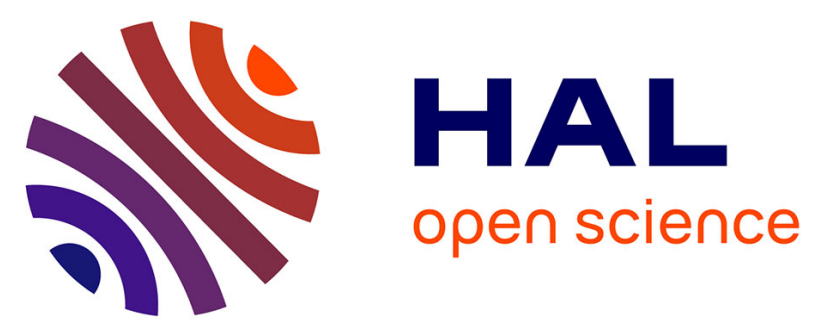

\title{
Valorisation professionnelle des biais attributifs commis par des candidats à un emploi confrontés aux potentialités d'inférences du Gros Cinq
}

Bernard Gangloff

\section{- To cite this version:}

Bernard Gangloff. Valorisation professionnelle des biais attributifs commis par des candidats à un emploi confrontés aux potentialités d'inférences du Gros Cinq. Psychologie du travail et des organisations, 2009, 15 (3), pp.229-246. 10.1016/S1420-2530(16)30171-6 . hal-01052878

\section{HAL Id: hal-01052878}

\section{https://hal-univ-paris8.archives-ouvertes.fr/hal-01052878}

Submitted on 7 Feb 2018

HAL is a multi-disciplinary open access archive for the deposit and dissemination of scientific research documents, whether they are published or not. The documents may come from teaching and research institutions in France or abroad, or from public or private research centers.
L'archive ouverte pluridisciplinaire HAL, est destinée au dépôt et à la diffusion de documents scientifiques de niveau recherche, publiés ou non, émanant des établissements d'enseignement et de recherche français ou étrangers, des laboratoires publics ou privés. 


\title{
Valorisation professionnelle des biais attributifs commis par des candidats à un emploi confrontés aux potentialités d'ỉnférences du Gros Cinq ${ }^{1}$
}

\author{
Professional valorization of attributive biases commited by \\ aplicants for a job confronted to the Big Five potential inferences \\ Bernard GANGLOFF \\ Département de Psychologie, Université de Rouen (France). \\ bernard.gangloff@univ-rouen.fr
}

\begin{abstract}
Résumé
Il est dhusage, en situation de recrutement, de caracteriser les candidats à un emploi par cing dimensions dites de personnalite. Pour ce faire, on utilise fréquemment des tests psychotechniques dans lesquels les réponses foumies donnent systénatiquement lieu à des attributions, et plus preciserment à des attributions internes alors mêtre qu'elles pouraient tout autant être In conséquence de facteurs causaux extemes. Les questions que nous.nous posons. ici portent, chme part sur róventuelle valorisation de ces infórences internes, et d'autre part, plus largement, sur réventuelle valorisation de lactivité attributive. Opérationnellement, nous avons demandé à des professionnels du recrutement d'indiquer, face atox items d'un questionnaire de big five exposant differentes conchutites, les attributions qu'tils attendraient d'un bon (yersus dum masvais).candidat a un emploi, sachant que manquaient les informations permettant d'effectuer une quelconque atribution de manière ratiomelle. Nous observons alors d'une part que la valorisation des atributions internes n'est pas systérmatique et qu'elle dépend notamment de la valence des items proposts, mais surtout que certaines attributions sont valorisées alors que notre questionnaire rendait toute attribution rationnellement impossible.
\end{abstract}

\section{Abstract}

it is of use, in recruiting situation, to characterize the applicants for a job by five dimensions known as personality dimensions. So one frequently uses the psychotechmical tests in which the given answers systematically conduce to assignments, and more precisely to imternal assignment, even if they could be often the consequence of external causal fictors. Our actual questions are, on the one hand about the possible valorization of these internal inferences, and on the other hand, thore extensively, about the possible valorization of the attributive activity. Operationally, we asked to professionals of the recruitment to indicate, facing the items of a big five questionnaire exposing different conducts, the assignments that they should wait from a good (versus a bad) applicant for a job, knowing that missed the information permiting to do rationally: any assignment. We observe, on the one hand that the valorization of the internal assigmment is not systematic and that it depends notably on the proposed item valence, but especially that some assigmnents are valorized whereas our questionnaine made any attribution rationally impossible.

Mots cles : recrutement, big,five, hétúro-attribution, biais. Key words; recruitmean, big five, hetero-attribution, bias.

\footnotetext{
'Par contraste à tune certgine misère universitaire parfois localement auto-genéré, qu'il nous soit permis de remercier ici R. Hausbif, notre genereux sponsor et fort sympathique boucher de la rue d'Alsace (76000 Roven) sans le financement duquel cette f́tude n'zurait jamais pu ftre mené ât terme.
} 


\section{Introduction}

Il est d'usage, dans de nombreuses professions de la psychologie, de caractériser les individus par des traits de personnalité. C'est-d-dire que les professionnels considérés observent des comportements puis, de cette observation (directe ou à partir de réponses fournies à des questionnaires autodescriptifis), ils en inferent des traits de personnaitité qu'ils atribuent aux individus. Ils en infêrent plus précisément cinq traits de personmalité, puisqu'actuellement le modèle des traits personnologiques le plus utilisé est le modèle en cinq facteurs (le Big Five) ${ }^{2}$, modèle hypothétisant que la personnalité peut etre caracterisé par cinq dimensions : extraversion, amabilité, caractère consciencieux, stabilité émotionnelle et ouvesture d'esprit. Une telle pratique reflète done une posture idéologique se conerétisant par une exclusion de la prise en compte de tout facteut environnemental comme facteur causal ayant pu intervenir dans l'adoption des comportements examiness, au profit d'un accent portant sur la personnalité de lacteur" considéré comme seute source de ces comportements.

Selon Beauvois ${ }^{3}$, une telle absence de prise en compte des facteurs contextueís permet de déresponsabiliser l'environnement, notamment social, et donc de légitimer sa préservation de toute velléité de remise en cause en réservant toute éventuelle sanction au seul acteur ainsi responsabilisé : puisque l'environnement n'intervient en rien dans l'adoption des comportements de l'individu, celui-ci est à considérer comme seul responsable de ses actes ; il ne servirait donc à rien de songer a critiquer l'enviromement et l'indiviđu doit seul subir tes cons\&quences de ses actes. Mais cette attribution de ses comportements a l'acteur as dans le cadre dun examen psychotechnique dovant déboucher sur des décisions d'action vis-à-vis de l'individu testé, d'autres conséquences.

Il convient alors de rappeler ( $c f$. Beauvois 1990) que l'établissement d'un profil personnologique est un acte évaluatif (et non descriptif), et qu'un tel acte n'est jamais gratuit : il participe toujours d'une utilite, d'un objectif : celui d'obtenir des eléments permettant de mieux décider des conduites à adopter vis-á-vis de l'individu testé (par exemple convient-il ou non de l'embaucher). Cela signifie que cette décision d'action semble nécessiter, pour optimiser sa justification, ce profil personnologique ;

2 cf. par exemple, en témoignage de l'acceptation quasi-consensuelle de ce modele : Caprara et al. (1997, p13), Rolland (1994, p65), ou encore (cités par Pervin, 1994, p103) Digman (1990, p439, Widiger (1993, p82), ctc..

${ }^{3}$ Par exemple (Beauvois, 1976, pl4) : "Le rôle de l'environnement dans le déterminisme des conduites évacué du process óvaluatif, [celui-ci peut] ¿éboucher sur la sanction de l'individu". 
cette attribution personnologique. Il est en effet évident que si l'on veut se déresponsabiliser de nos conduites distributives de sanction (embauche ou refus d'embauche d'wn candidat) et donc réaliser légitimement des attributions externes en matière de Locus of Distribution (Cf. Gangloff, 2004) afin de se premunir contre toute éventuelle accusation d'arbitraire de nos décisions, lun des meilleurs moyens est de déclarer lindividu examiné responsable de ses comportements et donc responsable des sanctions que ces comportements sont susceptibles de générer. En d'autres termes, la légitimité de l'application de sanctions étant tributaire de la possibilité d'álaboration d'un profil psychologique, et cette élaboration étant conditionnée par la responsabilité de l'individu dans ses comportements, il importerait de commencer par déclarer lindividu responsable de ces comportements ; cette responsabilité permettrait alors, dans un deuxième temps, de pratiquer des attributions internes en matière de Locus Of Control, c'est-ä-dire permettraít de déclarer l'individu responsable des sanctions dont il sera l'objet.

Il apparaift cependant que l'accent ainsi mis sur l'acteur résulte parfois d'une attribution illégitime et constitue ce qu'au $19^{\mathrm{kme}}$ siecle Nietzsche (6d.1968), puis plus rócemment Ross (1977), ont appelé une "erreur fondamentale", avant que des auteurs comme Jellison et Green (1981) y voient, du fait de la fréquence de cette "erreur", davantage une norme qu'une erreur. Kelley (1967) a ainsi considéré que le comportement d'un individu peut Etre attribué certes à la personne, mais aussi parfois amx stimuli ou aux circonstances si l'on disposait d'informations complémentaires ayant trait à la distinctivité, à la consistance et au consensus quant au comportement observét. Bt Mc Arthur (1972) a vérifié expérimentalement cette hypothèse en montrant qu'on obtenait une attribution à la personne en associant distinctivité faible, consensus faible et consistance forte; qu'on obtenait une attribution au stimulus par l'association de distinctivité forte, consensus fort et consistance forte; et qu'on aboutissait dे une attribution aux circonstances lorsque l'on associait distinctivite forte et consistance faible (le consensus n'intervenant pas pour cette attribution).

- La distinctivits concerne la variation des réketions de l'acteur à des stimuli équivalents, la consistance renvoie d'une part à la consistance dans le temps et d'aurtre part a la consistance malgré la variation du contexte dans lequel l'acteur est en contact avec le stimisus, et le consensus réfere a l'identité des conduites réactives de différents acteurs. Pour reprendre lun des exemples foumi par Mc Arthur, si l'on veut comâtre les causes du rire du spectateur foce au clown, il convient de se demander a) si le spectateur rit aux pitreries de tout clown ou. seulement a celles de l'actuel (distinctivite), b) si son rire est ou non systématique face $z$ ce clown (consistance), et c) si tout spectateur rit en présence de ce clown (consensus). 
Nous avons, dans une étude antérieure (Gangloff et Pasquier, 2007) mis en rapport la théorie du Big Five et celle de Kelley, en proposant a 152 sujets (étudiants et érudiantes inscrits en $1^{\text {th }}$ année de psychologie) des conduites référant aux 5 dimensions du Big Five et en demandant à nos sujets, au vu d'informations complémentaires de distinctivité, consistance et consensus, de classer, de manière la phus objective, la plus rationnelle possible, ces conduites en 3 catígories: dues à la personne, dues au stimulus, ou dues atri circonstances. Nous avons alors notamment constaté que les attributions à la personne (c'est- $\mathbf{x}$-dire attributions internes) n'étaient systématiques que si les informations complémentaires associant distinctivité faible, consensus faible et consistance forte s'y prêtaient (dans le cas contraire, les attributions portaient systématiquement plus fréquemment sur le stimulus ou sur les circonstances, selon la combinaison des informations complémentaires, ce quelle que soit la dimension comportementale considérée et quel que soit le póle -positif ou négatif- de cette dimension ${ }^{5}$ ). Mais nous avons surtout remarqué que meme en l'absence de- telles "informations" les attributions n'etaient que minoritairement personnologiques, ce que nous avons expliqué par le fait que nos sujets avaient alors pour tâche de rópondre de la manière la plus logique possible, sans aucune exigence d'une eventuelle sanction à appliquer. A contrario, nous avons observé que lorsque l'application d'une telle sanction était explicitement stipulée dans la tâche, en l'occurrence une pénalité à infliger à un délinquant, les attributions étaient systématiquement internes (Hardy-Massard et Gangloff, 20075). Mais outre que cette dernière étude entrait dans le domaine, bien particulier; dur judiciaire, nous n'y avions pas mis en rapport les attributions avec la thérie de Kelley.

Nous avons voulu ici croiser certains éléments de chacune de ces deux dernières études, dans une tache de recrutement où une sanction (embauche/refus d'embauche) devait être appliquée, avec comme bases de décision la présentation de conduites de candidats renvoyant aux cinq dimensions du Big Five, conduites non contextuées par des informations précisant leur degré de consensus, consistance et distinctivité, et done conduites interdisant toute attribution rationnelle. Et nous nous sommes placés en amont de la sanction : nous'nous sommes demandés si' des

${ }^{3}$ Cest-a-dire que les memes attributions sont obtenues si, sur la dimension "amabilité" par exemple, l'acteur adopte un comportement aimable ou non aimable.

- Dans cette Etude, 8 scetnarios representant chacun une infraction étaient présentés, les sujets devant indiquer Io nivear.de senction qu'ils considéraient devoir etre appliqué au délinquant puis expliquer pourquoi. Il a alors eté remargué que ces explications étaient formulées en termes düntensité de responsabilité attribuée au délinquant. 
professionnels du recrutement et de l'insertion allaient, malgré cette impossibilité; valoriser les inférences attributives, et notamment si ils allaient vajoriser ies inférences permissives d'attributions personnologiques.

\section{Methode}

Nous avons utilisé "le paradigme du législateur" (Gangleff; 2006), c'est-à-dire que nous avons présenté à des professionnels du recrutement et de linsertion un questionnaire mettant en scène 10 comportements, à la suite de chacun desquels nos sujets devaient cocher, parmi trois attributions proposées (et non exclusives entre elles), d'une part celle(s) qu'ils souhaiteraient obtenir d'un bon candidat à un emploi, et d'autre part celle(s) qu'ils pensajent qu'ils obtiendraient d'un mauvais candidat?.

Le questionnaire utilise ${ }^{8}$ est constitué de 10 phrases (déjả antérieurement employées et validées : Gangloff et Pasquier, 2007) renvoyant aux 5 dimensions - du Big-Five (2 phrases par dimension; l'unesituée sur le p6le positif et la seconde sur le póle négatif). Par exemple, pour la dimension "amabilite", les 2 items suivants étaient utilisés : "Avec les membres de sa famille, Paul se montre agréable" (item renvoyant à la dimension "amabilité", formulé de manière positive) et "avec les membres de sa famille. Paul se montre désagréable" (item renvoyant à la dimension "amabilité", formulé de manière négative)". A la suite de chacum des 10 items, le sujet devait cocher une (ou plusieurs) proposition(s) parmi trois correspondant respectivement à une attribution $a$ la personme (proposition $A$ ), aut stimulus (proposition $B$ ) et aux cireonstances (proposition .C). Par exemple, pour litem "amabilité" formulé positivement, les trois attributions suivantes étaient proposées : "A-C'est en lui, c'est dans sa nature de se montrer agréable", "B-Ce

${ }^{7}$ Precisons que rien n'oblige formellement les sujets a fournir des réponses différentes pour lo bon candidat et pour lo mauvais, ou plus précisément ì crér des différences qu'lis n'auraient pas spontanfinent envisagés. Le paradigme que nous avons utilisé se rappuoche donc du paradigme du juge avec passations intraindividuelles. Rappelons par ailleurs qu'vne etude antórieure (Gangloff 2003) avait mis en évidence l'absence d'impact du poste à pouvoir et du secteur d'activité (publichprive) sur le profil attendu d'un bon candidat. Nous avons donc choisi ici de ne specifier ni poste ni secteur, privilgiant ainsi l'extension eventuelle des résuitats que nous pouvions obtenir.

Sa consigne et sa presentation complete figurent en atnexe.

- A l'instar de ce que l'on observe dans. les inventaires, de personnalitio, ot. plus globalement dans les situations de recrutement, nous avons choisi de mettre en scène des situations qui relèvent des différentes sphères de vie et pas uniquement de la sphère professionnelle. 
sont les membres de sa famille qui induisent qu'tl se montre agréable", ${ }^{\circ} \mathrm{C}$ - Ce sont des circonstances particulières qui l'amènent à se montrer agréable". Nous avons alors étudié la répartition des réponses fournies à chaque item, quel que soit le nombre de ces réponses, ${ }^{10}$, et nous y avons appliqué des traitements par $\mathrm{X}^{2}$. Au cas où nos sujets tiendraient bien compto de l'impossibilité d'effectuer rationnellement une quelconque attribution, nous ne devrions obtenir aucune valorisation (aucune case ne devrait être cochée). Dans le cas contraire, nous voulions savoir si les valorisations ne portaient pas davantage sur les attributions personnologiques que sur les autres, et si la dimension examinée ou/et son pôle (positif ou négatif) modulaient les valorisations obtenues.

Notre échantillon est constitué de 21 sujets ( 5 hommes et 16 femmes), tous specialistes des ressources humaines (travaillant dans le recrutement, l'orientation, et l'insertion professionnelle). Ils se répartissent, quant à leur age, entre 25 et 55 ans (12 ont entre 25 et 35 ans, 6 entre 35 et 45 ans, 2 entre 45 et 55 , et 1 a plus de 55 ans). Enfin, 4 font état d'une expérience professionnelle de leur fonction -inférieure i 1 an, 4 ont une expérience. comprise entre 1 et 5 ans, et 13 ont une expérience supérieure à 5 ans.

\section{Résultats}

Est consignée dans le tableau 1 la répartition des réponses toutes. dimensions confondues. Les analyses statistiques réalisées conduisent aux principaux constats suivants.

1) En considérant les pôles positifs et négatifs ensemble, aucune différence significative n'epparaît entre les 3 attributions, ni en ce qui concerne les réponses attendues du bon candidat ni pour celles du mauvais candidat. Malgré cette équivalence de répartition entre les 3 attributions, on observe que les attributions aux circonstances sont plus frequentes pour les réponses attendues du bon candidat par rapport à celles escompttes du mauvais candidat $\left(X^{2}=15,37 ; p<0,001\right)$.

2) Au niveau des póles positifs, on remarque que pour les bons candidats les attributions à la personne sont plus fréquentes que celles au stimulus $\left(X^{2}=10,94 ; p<0,001\right)$ ou aux circonstances $\left(X^{2}=4,83 ; p=0,03\right)$. Inversement, pour les mauvais candidats, les attributions à la personne sont moins fréquentes que celles au stimulus ou aux circonstances (avec dans les 2 cas $\mathrm{p}<0,001$ ). Les comparaisons des réponses attendues du bon et da mauvais candidat montrent également que les attributions à la

\footnotetext{
10 Nous avons en effet considáré que nos sujets pouvajent tọut aussi bien exprimer valablement leurs choix en ge cochant aucune case (si aucune des trois propositions fournies ne correspondait aux réponsses qu'ils expectaient d'un "bon" ou d'un "mauvais" candidat), qu'en en cochent une, deux, voire les trois.
} 
personne sont plus fréquentes pour les bons candidats que pour les mauvais $(p<0,001)$, de même que les attributions aux circonstances $(\mathrm{p}=0,04)$.

3) Pour les pôles négatifs, les résultats sont pratiquement inverses. Ainsi les bons candidats sont censés faire moins d'attributions à la personne qu'au stimulus ou aux circonstances (dans les 2 cas avec $p<0,001$ ), alors que les mauvais candidats sont dits attribuer davantage à la personne qu'au stimulus. $(p=0 ; 003)$ ou aux circonstances $(p<0,001)$; On constate également que les bons candidats font moins d'attributions à la personne $(p<0,001)$ et davantage d'attributions aux circonstances $(p<0,001)$ que les maurvais.

4) On remarque enfin, en comparant les póles positifs et neggatifs, que chez les bons candidats les attributions a la personne sont plus. fréquentes en situations positives qu'en situations négatives $(p<0,001)$ alors que les données sont inverses pour les mauvais candidats $(p<0,001)$.

Tableau $I$ i repartition des atributions, les 5 dimensions confondîtes. NB : rappelöns que nas sujets pouvaient, a chacun des 10 items, et aussi bien pour le "bon" que pour le "manvats" candidat, ne cocher aucune case, ou en cocher 1, 2 ou 3, Cela explique que les sommes horizontales pour un "bon" et pour wn "mavwas" candidat peuwent Etre differentes cussi bien entre elles que d'une ligne d'autre (il en est de weline au tableau 2).

\begin{tabular}{lccccccc}
\hline & \multicolumn{3}{c}{ Bon candidat } & \multicolumn{3}{c}{ Mauvais candidat } \\
\cline { 2 - 7 } & $\begin{array}{c}\text { Atrib. } \\
\text { Pers. }\end{array}$ & $\begin{array}{c}\text { Attrib. } \\
\text { Stim. }\end{array}$ & $\begin{array}{c}\text { Atrib. } \\
\text { Circ. }\end{array}$ & $\begin{array}{c}\text { Attrib. } \\
\text { Pers }\end{array}$ & $\begin{array}{c}\text { Attrib. } \\
\text { Stim. }\end{array}$ & $\begin{array}{c}\text { Attrib. } \\
\text { Circ. }\end{array}$ \\
\hline $\begin{array}{l}\text { Somme pôles positifs,et. } \\
\text { négatifs groupśs }\end{array}$ & 102 & 94 & 130 & 99 & 98 & 74 \\
\hline $\begin{array}{l}\text { Somme póles positifs } \\
\text { Somme pôles négatifs }\end{array}$ & 89 & 50 & 62 & 13 & 47 & 41 \\
\hline
\end{tabular}

Si l'on analyse maintenant les résultats de manière plus fine, c'est-ddire dimension par dimension, on aboutit au tableau 2. 
Tableau 2 : repartition des attributions en fonction des dimensions et des poles pasitifisindzatifs.

\begin{tabular}{|c|c|c|c|c|c|c|c|}
\hline & & \multicolumn{3}{|c|}{ Bon candidat } & \multicolumn{3}{|c|}{ Mauvais candidat } \\
\hline & & $\begin{array}{l}\text { Attrib. } \\
\text { Pers. }\end{array}$ & $\begin{array}{l}\text { Attrib. } \\
\text { Stim. }\end{array}$ & $\begin{array}{l}\text { Attrib } \\
\text {. Circ. }\end{array}$ & $\begin{array}{l}\text { Attrib. } \\
\text { Pers. }\end{array}$ & $\begin{array}{l}\text { Attrib } \\
\text {.Stim. }\end{array}$ & $\begin{array}{l}\text { Attrib. } \\
\text { Circ. }\end{array}$ \\
\hline \multirow[t]{2}{*}{ Extraversion } & Póle posit. & 17 & 5 & 15 & 2 & 15 & 5 \\
\hline & Pôle négat. & 3 & 9 & 15 & 18 & 8 & 4 \\
\hline \multirow[t]{2}{*}{ Amabilité } & Pôle posit. & 17 & $1 \mathrm{I}$ & 10 & 4 & 7 & 11 \\
\hline & Pôle négat. & 1 & 9 & 14 & 20 & 9 & 6 \\
\hline \multirow{2}{*}{$\begin{array}{l}\text { Caractère } \\
\text { Consciencieux }\end{array}$} & Pôle posit. & 17 & 17 & 13 & 4 & 3 & 7 \\
\hline & Pôle négat. & 2 & 10 & 11 & 15 & 11 & 10 \\
\hline \multirow{2}{*}{$\begin{array}{l}\text { Stabilité } \\
\text { émotionnelle }\end{array}$} & Póle posit. & 18 & 11 & 12 & 2 & 9 & 8 \\
\hline & Pôle négat. & 5 & 12 & 16 & 16 & 8 & 4 \\
\hline \multirow{2}{*}{$\begin{array}{l}\text { Ouverture } \\
\text { d'esprit }\end{array}$} & Pôle posit. & 20 & 6 & 12 & 1 & 13 & 10 \\
\hline & Póle negat. & 2 & 4 & 12 & 17 & 15 & 9 \\
\hline
\end{tabular}

Les analyses statistiques réalisées sur ce tableau 2 meftent alors en évidence de nombreuses différences significatives (avec $p$ toujours au moins inférieur a 0,05 ).

1) Pour l'extraversion

- considérte positivement tout d'abord, les bons candidats fournissent davantage de réponses impliquant la personne que de réponses liées au stimulus $\left(X^{2}=6,55 ; p=0,01\right)$, l'inverse étant observé chez les marvais candidats $\left(X^{2}=9,94 ; p=0,001\right)$, ce qui conduit à plus d'attributions à la personne $\left(X^{2}=11,84 ; p<0,001\right)$ et à moins d'attributions ar stimulus $\left(X^{2}=5,00 ; p=0,02\right)$ chez les bons candidats que chez les mauvais ;

sur le pôle négatif maintenant, les bors candidats réalisent plus d'attributions aux circonstances qu'à la personne, et les mauvais plus d'attributions a la personne qu'au stimulus ou aux circonstances, soit, en comparant les bons aux mauvais candidats, moins d'attributions à la personne et plus d'attributions aux circonstances chez les bons que chez les mauvais. 
2) Pour l'amabilité

- considérée positivement, une seule différence significative apparaît, avec davantage d'attributions a la personne chez les bons candidats que chez les mauvais $\left(X^{2}=8,05 ; p=0,004\right)$;

sur le pôle négatif, on remarque, chez les bons candidats plus d'attributions au stimulus ou aux circonstances qu'à la personne, les đonnées des manvais candidats étanî diamérralement opposées, soit, en comparant les bons aux mauvais, moins d'attributions à la personne chez les bons que chez les marvais candidats.

3) Pour Ia conscience professionnelle

- sur le pôle positif, on remarque davantage d'attributions à la personne et au stimulus chez les bons candidats que chez les mauvais ;

sur le póle négatif, les bons candidats effectuent plus dattributions an stimulus ou aux circonstances qu'b la personne, et réalisent moins d'attributions à la personne que les mauvais candidats.

4) Pour la stabilitit émotionnefle

- sur le póle positif, les mauvais candidats attribuent davantage au stimulus et aux circonstances qu'a la personne, et corollairement les bons candidats attribuent plus à la personne que les mauvais ;

sur le pôle négatif, les bons candidats attribuent plus aux circonstances qu'à la personne, les mauvais présentant un profil diamétralement opposé, et plus globalement les bons candidats font, par rapport aux mauvais, moins d'attributions à la personne et plus aux circonstances.

5) Pour Houverture d'esprit

* sur le pôle positif, les bons candidats font davantage d'attributions à la personne qu'au stimulus et les mauvais davantage au stimulus ou aux circonstances qu'à la personne, ce qui aboutit à plus d'attributions a la personne chez les bons que chez les mauvais candidats ;

sur le pôle négatif, les bons candidats attribuent plus aux circonstances qu'à la personne, et plus globalement moins d'attributions a la personne ou au stimulus que les mauvais candidats.

Ces différents résultats sont synthétisés dans le tableau 3 suivant. 
Tableau 3 : différences significativives dans les répartitions des attributions (avecp systénatiquement ou moins inferieur ì 0,05).

$N B$ : les inégalites non significatives ne sont pas reproduites dans le tablean.

\begin{tabular}{|c|c|c|c|c|}
\hline & & $\begin{array}{c}\text { Bons } \\
\text { candidats }\end{array}$ & $\begin{array}{l}\text { Mauvais } \\
\text { candidats }\end{array}$ & Bons/Mnuvais \\
\hline \multirow{2}{*}{ Extraversion } & Pole posit. & $P>S$ & $P \propto S$ & $\begin{array}{l}\text { Plus de } \mathbf{P} \text { et moins de } \mathbf{S} \\
\text { chez les bons }\end{array}$ \\
\hline & Pôle négat. & $\mathrm{CP}$ & $P>S$ et $P>C$ & $\begin{array}{l}\text { Moins de } \mathrm{P} \text { et plus de C } \\
\text { chez les bons }\end{array}$ \\
\hline \multirow{2}{*}{ Amebilite } & POle posit. & & & $\begin{array}{c}\text { Plus de P } \\
\text { chez les bons } \\
\end{array}$ \\
\hline & Póle négat & $\mathrm{SP}$ et $\mathrm{C}>\mathrm{P}$ & $\mathrm{P}>\mathrm{S}$ et $\mathrm{P}>\mathrm{C}$ & $\begin{array}{c}\text { Moins de } P \\
\text { chez les bons }\end{array}$ \\
\hline \multirow{2}{*}{$\begin{array}{c}\text { Caractère } \\
\text { Consciencieux }\end{array}$} & P6le posit. & & & $\begin{array}{c}\text { Plus de } P \text { et plus de } S \\
\text { chez les bons }\end{array}$ \\
\hline & Pôle négat. & $\mathrm{SP}$ et $\mathrm{C}>\mathrm{P}$ & & $\begin{array}{c}\text { Moins de } \mathbf{P} \\
\text { chez les bons }\end{array}$ \\
\hline \multirow{2}{*}{$\begin{array}{l}\text { Stabilite } \\
\text { emotionnelle }\end{array}$} & Póle posit & & SP et C >P & Plus de $P$ chez les boos \\
\hline & Pole nfgat. & CPP & $\mathrm{P}>\mathrm{C}$ & $\begin{array}{l}\text { Moins de P et plus de C } \\
\text { chez les bons }\end{array}$ \\
\hline \multirow{2}{*}{$\begin{array}{l}\text { Ouverture } \\
\text { desprit }\end{array}$} & Pole posit. & P>S & S9 et C >P & $\begin{array}{c}\text { Plus de } P \\
\text { chez les bons }\end{array}$ \\
\hline & Pôle nógat. & $\mathbf{O P}$ & & $\begin{array}{l}\text { Moins de } \mathrm{P} \text { ot moins de } \mathrm{S} \\
\text { chez les bons }\end{array}$ \\
\hline
\end{tabular}

\section{Discussion et conclusion}

Le principal constat de cette recherche est une nette spécification du profil souhaite (et, corollairement, du profil rejete), mais specification qui se traduit de manidre différencí́e selon le pỏle positif versus négatif des dimensions. Le bon candidat est ainsi quelqu'un qui, pour les póles positifs, fait état d'attributions plus fréquentes à la personne qu'av stimulus ou aux circonstances, alors que le muuvais candidat réalise des attributions inverses. En revanche, pour les pôles négatifs, il est attendu des bons candjdats qu'ils fassent davartage appel aur stimulus ou aux eirconstances qu'a la personne (avec des attentes inverses vis-à-vis des mauvais candidats). Synthétiquement parlant, on aftend donc que lo bon candidat fasse (par rapport au mauvais) plus d'attributions internes sur les pôles positifs des dimensions, et plus d'attributions externes (au stimulus ou aux circonstances) sur les pôles négatifs. Par ailleurs, autre constat non totalement dénué d'intérêt, si l'on remarque bien que ces résultats 
sont globalement valables quelle que soit la dimension considérée, on observe cependant que certaines dimensions font l'objet d'attentes plus intenses que d'autres. Ainsi, pour les dimensions "amabilite" et "caractère consciencieux" considérées positivement, il n'est pas attendu des bons candidats qu'ils attribuent davantage à la personne, ni des mauvais candidats qu'ils attribuent davantage au stimulus ou aux circonstances (ce qui contraste notmmnent par rapport gux dimensions "extraversion" et. "ouverture d'esprit"). On remarque enfin, sur un plan plus accessoire, que les pôles négatifí conduisent à davantage d'attentes attributives que les poles positifs.

Commençons alors par rappeler qu'il est souvent considéré que les attributions internes sont systématiquement valorisées, au point que l'on parle de norme d'internalité. Et le principal argument avancé pour étayer cette éventuelle existence d'une norme d'internalité est que cette norme permettrait arx agents de pouvoir de justifier d'une sanction à appliquer à l'objet examiné (comme par exemple de légitimer une décision d'embauche ou de refus d'embauche : cf. par exemple Beauvois; 1984, p118 et s.). De nombreuses travaux, tant français qu'étrangers, ont cependant déjà mis en évidence que l'internalité n'est valorisóe que si elle ne remet pas en cause la hiérarchie des pouvoirs dans laquelle elle s'inscrit, c'est-d-dire que si elle se concrétise sous une forme allégeante (cf. par exemple Gangloff 2002). Nous observons maintenant une nouvelle limite a la valorisation des explications internes puisque cette valorisation n'est ici effective que lorsqu'elle porte sur des conduites positives : dans le cas de conduites négatives, ce sont les explications externes, en termes de stimulus ou de circonstances, qui sont plébiscitses. Nous observons donc à nouveau que la valorisation de l'internalite n'est pas systénatique, et que cette absence de systématisme n'oblitłre ici aucunement liapplication d'une sanction.

Sur un autre plan, il convient de souligner que le questionnaire ici utilisé ne contenait aucune information contextuelle permettant une attribution objective des causes des conduites décrites. Cela signifie que tout observateur rationnel de ces conduites était placé dans l'impossibilité de réaliser une quelconque attribution, ce qui aurait du se traduire par l'absence de toute valorisation attributive de la part de nos sujets. Or il est observé que les professionnels interrogés attendent d'autres réactions, réactions se traduisant par des attributions différenciées. En d'autres termes, nos sujets attendent des candidats à un emploi qu'ils se comportent de manière irrationnelle et commettent des biais attributifis : biais de complaisance (Zuckerman, 1979) ou de positivité (Lewicka, Czapinski et Peters, 1992) vis-hi-vis des bons candidats dont on sollicite. qu'ils expliquent les conduites positives par des attributions à la personne et dont on attend qu'ils expliquent les conduites négatives en réalisant 
davantage d'attributions externes qu'internes (ce que l'on pourrait considérer comme renvoyant à un biais de "déresponsabilisation"). Pour autant, tous les biais ne sont pas vaiorisés. Ainsi les mauvais candidats sont ceux qui, privilegiant le stimulus ou les circonstances pour expliquer les conduites positives, appliquent le biais de modestie ( $c f$. Dubois, 1994, p101), ou qui, expliquant les conduites négatives par des attributions intemes font en fait appol to un biais que l'on pourrait nommer de "mortification"!'. Puisque tous les biais me sont.pas valorises, il importe donc de s'interroger un instant sur les critères susceptibles de différencier les bons biais des mauvais. Rappelons alors, pour reprendre la dichotomisation de Weiner et col. (1972), que les attributions intemes que nous avons proposées dans notre questionnaires sont des attributions stables, alors que celles réförant aux stimuli et aux circonstances sont à considérer comme instables. Si l'on applique done ici la dichotomie de Weiner, on aboutit à une valorisation des biais reliant les bonnes conduites (i.e. les pôles positifs des dimensions) à des caractéristiques issues de la personne et stables, et les marrvistes à des phénomènes instables, environnementaux.

Plus fondamentalement, nous venons de dire que nos sujets valorisent les candidats realisant des inférences attributives biaisés. Or la valorisation d'un biais constítue l'un des éléments permettant de passer du statut de biais à celui de norme. Cest par exemple ainsi, suite à une étude de Jellison et Green (1981), que le bizis d'internalité a été abandonné au profit du concept (hypothétique) de norme d'intemalité. Il est donc possible de se demander si la valorisation des biais que nous venons d'observer n'est pas; elle aussi, susceptible de conforer a ces dits biais le statut de normes ; mais bien évidemment, pour le confirmer, des études complémentaires sont nécessaires... Autre point à étudier : quelles sont les attentes des recniteurs lorsque les conduites à expliquer sont accompagnées d'informations contextuelles impliquant objectivement des attributions a la personne, au stimulus, ou aux circonstances? Nous n'avons, dans la présente étude, fourni aucune de ces informations (d'où l'inpossibilité de réaliser, rationnellement, une quelconque attribution). Or selon la thérie de Kelley, que nous avons rappelée en introduction, l'association de distinctivité faible, consensus faible et consistance forte doit conduire à une attribution à la personne ; l'association de distinctivité forte, consensus fort et consistance forte doit aboutir \& une attribution au stimulus; et on doit obtenir une attribution aux circonstances lorsque l'on associe distinctivité forte. et consistance faible. La question à examiner

\footnotetext{
"Ce reflus des biais de madestie et de "mortifioation" ne sigalifie évidemment pas que l'on rejeette los marvivis condidats en raison de leurs attributions biaistes (et qu'dolors on attendrait d'eux qu'ils se comportent de manietre plus rationnelle), mais seulement que leur stigmatisation provient de lenr non application des bons biais, c'est-d-dire des biais de compledsance et de "deresponsabilisation"
} 
serait donc de savoir sì, disposant de ces informations et réalisant les inférences qui logiquement en résultent, les candidats feront l'objet d'une valorisation. Si ce n'est pas le cas, cela signifierait globalement, non seulement une valorisation de réactions attributives alors qu'aucune réaction n'est rationnellement permise (ce que nous observons ici), mais également une bien curieuse faveur octroý́e aux candidats réalisant des attributions erronées plutôt qu'exactes. Et il conviendrait alors de s'interroger sur les objectifs, voire sur les idéologies, à la base de ces attentes. Comme on le constate, de nombreuses pistes de recherches sont encore a explorer. Mais bien évidemment, tout ceci ne constitue que des hypothèses, et si elles se révélaient exactes, le rôle de la science (sauf à s'exposer aux rigoureux jugements de valeur de l'jdéologie académique) ne serait que d'en rapporter le constat, sans entrer dans un jugement de valeur qui re pourrait être qu'idéologique. En attendant donc la mise à l'épreuve do ces hypothèses, nous espérons que les présents rúsultats participeront à nourrir les réflexions des praticiens quant à l'utilisation des réponses qu'its obtiennent aux inventaires de personnalité qu'ils-sent susceptibles d’appliquer.

\section{References}

Beauvois, J-L. (1976). Problématique des conduites sociales d'Evaluation. Conmexions, 19, 7-30.

Beauvois, J-L. (1984), La Psychologie quotidienne. Paris : PUF

Bearvois, J-L. (1990). L'acceptabilité sociale et la connaissance Evaluative. Connexions; 59; 7-16:

Caprars, G.V., Barbaranellt, C. et Borgogni, L. (1997). Alter ego ; les I facteurs fondamentanox de la personnalite. Paris : E.A.P.

Dubois, N. (1994). La norme d'internalité et le libéralisme. PUG

Gangloff, B. (2002). L'internalité et l'allégeance considérées comme des normes sociales. Les Cahiers de Psychologie Politique, $\mathrm{n}^{\circ} 2$. [http://www.cahierspsypol.fi].

Gangloff, B. (2003)، D'une nature persomologigue a une nature normative des 5 facteurs du Big Five. Les Cahiers de Psychologie Politique, $\mathrm{n}^{\circ} 4$. [http://www,cahierspsypol.fi].

Gangloff, B' "(2004); Le parapluie de Ponce Pilate, ou la valorisation de l'externalites en matiers d'explication des comportements distributifs de sanetions (Locus of Distribution). Psychologie du Travail et des Organisations, vol.10(4), 313-326.

Gangloff, B. (2006). Normativite de la croyance en un monde juste via "le paradigme du legislateur" : une étude sur des recruteurs. 1 f $^{\text {mat }}$ Congres International de Psychologie du Travail de Langue Française. Hammamet (Tunisie). 
Gangloff, B. et Pasquier, D. (2007). When personality traits psychology preserves the social order : an implementation on the Big Five theory via. the attributional cube theory of Kelley. $5^{\text {th }}$ Intematio nel Conference of Applied Psychology. Timisoara (Roumanie).

Hardy-Massard, S. et Gangloff, B. (2007), Attributional biases in the judicial decisions. $5^{\text {th }}$ International Conference of Applied Psychology. Timisoara (Roumanie).

Jellison, JM. et Green, J. (19:1). A self-presentation approache to the findamentat atribution error : the norm of internality. Journal of Personality and Social Psychology, 40, 4, 643-649.

Kelley, H.H. (1967). Attribution theory in social psychology. In : L. Levine (Ed). Nebrasika Sympasium on Motivation Lincoln : University of Nebraska Press, 15, 192-238.

Lewicka, M., Czapinaki, J. et Peters, G. (1992). Positive-negative asymetry or when the heart needs a reason, Exropeon Joumal of Soctal Psychology, 22, 425-434.

Mc Arthur, L. (1972). The how and what of why : some determinants and consequences of causal attribution. Joumal of Personaliny and Social Psychologi, 22, 172-193:

Nietzsche, F. (ed. franc. 1968). Humain trop humain. Paris : Gallimard, vol.1

Pervin, LA. (1994). A critical analysis of current trait theory. Psychological Inquiny, 5(2), 103-113.

Rolland, J-P. (1994). Dessirabilité sociale dz marqueurs des dimensions de la persotnalitie du modale en 5 facteurs. Revue Europeame de Psychologile Appliqué, 4(1), 65-71.

Ross, L. (1977). The intuitive psychologist and his shortcomins : distorsions in the attribution process. In : L. Berkowitz (Ed.). Advances in experimental social psychology. New-York : Academic Press, vol.10, 173-220.

Weiner, B., Friezs, I., Kulkle, A., Reed, L., rest, S. et Rosenbaum, R-M. (1972), Perceiving the caurses of success and failure. In : E.E. Jones, D.E. Kanouse, H.H. Kelley, RE. Nisbett, S. Valins et B. Weiner (Eds). Attribution : perceiving the causes of beavior. Morristown : General learning Press.

Zuckerman, M. (1979). Atribution of success and failure revisited, or : the motivational bias is alive and well in attribution theory. Joumal of Personality and Social Psychology, 47, 245-287. 


\section{Annere consigne et contenu da questionnaire utilise ${ }^{12}$}

Dans le questionnaire suivant vouss sont présentées 10 phrases quj décrivent le comportement d'un personnage, ce comportement étant suivi de 3 causes.

II vous est demande d'indïquer, face à chacune de ces propositions : - les causes que vous aimeriez obtenir d'un bon candidat à un emploi, les causes que donnerait, selon vous, un mauvais candidat a un emploi.

\begin{tabular}{|c|c|c|}
\hline & $\underset{\text { candidat }}{\text { Bon }}$ & $\begin{array}{l}\text { Mauvais } \\
\text { candidat }\end{array}$ \\
\hline \multicolumn{3}{|l|}{ 1. Dans cette acthide, Paul se montre minutleux. } \\
\hline $\begin{array}{l}\text { A- C'est en lui, c'est daws sa nature de se } \\
\text { montrer minutieux. }\end{array}$ & & \\
\hline $\begin{array}{l}\text { B- C'est cette activité qui indút qu'il se montre } \\
\text { minutieux. }\end{array}$ & & \\
\hline $\begin{array}{l}\text { C-Ce sont des circonstances particulières qui } \\
\text { l'amènent â se montrer minutfelux. }\end{array}$ & & \\
\hline
\end{tabular}

\begin{tabular}{|c|c|c|}
\hline & $\begin{array}{c}\text { Bon } \\
\text { candidat }\end{array}$ & $\begin{array}{l}\text { Mëuvalis } \\
\text { candidat }\end{array}$ \\
\hline 2. Fn cas de difficulté, Paul se montre inquiet. & & \\
\hline $\begin{array}{l}\text { A- C'est en lui, c'est dans sa nature de se montrer } \\
\text { inquiet }\end{array}$ & & \\
\hline $\begin{array}{l}\text { B- C'est la difficulté qui indujt qu'il se montre } \\
\text { inquiet. }\end{array}$ & & \\
\hline $\begin{array}{l}\text { C- Ce sont des circonstances particulières qui } \\
\text { l'amdnent ì se montrer inquiet. }\end{array}$ & & \\
\hline
\end{tabular}

\footnotetext{
${ }^{12} L$ 'extraversion est presentee, dans ses poles respectivement positif et négatif, aux items 10 et 5, l'amabilité aux items 3 et 8, le caractère consciencieux aux items 1 et 6, la stabilité enotionealle aux items 7 et 2 , et l'ouverture d'esprit aux iterns 4 et 9.
} 


\begin{tabular}{|c|c|c|}
\hline & $\begin{array}{c}\text { Bon } \\
\text { candidat }\end{array}$ & $\begin{array}{l}\text { Mauvais } \\
\text { candidat }\end{array}$ \\
\hline \multicolumn{3}{|c|}{ 3. Avec les membres de sa famille, Paul se montre agréable. } \\
\hline $\begin{array}{l}\text { A- C'est en lui, c'est dans sa nature de se } \\
\text { montrer agréable. }\end{array}$ & & \\
\hline $\begin{array}{l}\text { B-Ce sont les membres de sa famille qui.. } \\
\text { induisent qu'il se montre agréable. }\end{array}$ & & \\
\hline $\begin{array}{l}\text { C- Ce sont des circonstances particulières } \\
\text { qui l'amènent à se montrer agreable. }\end{array}$ & & \\
\hline
\end{tabular}

\begin{tabular}{|l|l|l|}
\cline { 2 - 3 } \multicolumn{1}{l|}{} & $\begin{array}{c}\text { Bon } \\
\text { candidat }\end{array}$ & $\begin{array}{c}\text { Mauvais } \\
\text { candidat }\end{array}$ \\
A- Avec ses anis, Paul se montre targe d'esprit. & & \\
montrer large d'esprit. & & \\
B-Ce sont ses amis qui induísent qu'il se & & \\
montre large d'esprit. & & \\
C- Ce sont des circonstances pgrticulières & & \\
qui l'amènent à se montrer large d'esprit. & & \\
\hline
\end{tabular}

\begin{tabular}{|c|c|c|}
\hline & $\begin{array}{c}\text { Bon } \\
\text { candidat }\end{array}$ & $\begin{array}{l}\text { Manvais } \\
\text { candidat }\end{array}$ \\
\hline \multicolumn{3}{|c|}{ 5. Frace d des inconnus, Paul se montre peu sâr de lut. } \\
\hline \multicolumn{3}{|l|}{$\begin{array}{l}\text { A- C'est en lui, c'est dans sa nature de se } \\
\text { montrer peu sâr de lui. }\end{array}$} \\
\hline \multicolumn{3}{|l|}{$\begin{array}{l}\text { B-Ce sont les inconnus qui induisent qu'il se } \\
\text { montre peu sur de lui. }\end{array}$} \\
\hline $\begin{array}{l}\text { C- Ce sont des circonstances particulières } \\
\text { qui l'amenent à se montrer pez sâr de luí. }\end{array}$ & & \\
\hline
\end{tabular}




\begin{tabular}{|c|c|c|}
\hline & $\begin{array}{c}\text { Bon } \\
\text { candidat }\end{array}$ & $\begin{array}{l}\text { Mauvais } \\
\text { candidat }\end{array}$ \\
\hline \multicolumn{3}{|c|}{ 6. Dans cette activite, Paul se montre peu minutieux. } \\
\hline \multicolumn{3}{|l|}{$\begin{array}{l}\text { A- C'est en lui, c'est dans sa nature de se } \\
\text { montrer peu minutieux. }\end{array}$} \\
\hline \multicolumn{3}{|l|}{$\begin{array}{l}\text { B- C'est cette activité qui induit qu'il se } \\
\text { montre peu minutieux. }\end{array}$} \\
\hline $\begin{array}{l}\text { C-Ce sont des circonstances particulières } \\
\text { qui l'amènent à se montrer peu } \\
\text { minutieux. }\end{array}$ & & \\
\hline
\end{tabular}

\begin{tabular}{|c|c|c|}
\hline & $\begin{array}{c}\text { Bon } \\
\text { candídat }\end{array}$ & $\begin{array}{l}\text { Mauvais } \\
\text { candidat }\end{array}$ \\
\hline \multicolumn{3}{|l|}{ 7. En cas de difficulte, Paul se montre calme. } \\
\hline \multicolumn{3}{|l|}{$\begin{array}{l}\text { A-C'est en lui, c'est dans sa nature de se } \\
\text { montrer calme. }\end{array}$} \\
\hline \multicolumn{3}{|l|}{$\begin{array}{l}\text { B-C'est la difficulté qui. induit qu'il se } \\
\text { montre calme. }\end{array}$} \\
\hline $\begin{array}{l}\text { C- Ce sont des circonstances particulières } \\
\text { qui l'amènent ì se montret calme. }\end{array}$ & & \\
\hline
\end{tabular}

\begin{tabular}{|l|l|l|}
\cline { 2 - 3 } \multicolumn{1}{l|}{} & \multicolumn{1}{c|}{$\begin{array}{c}\text { Bon } \\
\text { candidat }\end{array}$} & $\begin{array}{l}\text { Mauvais } \\
\text { candidat }\end{array}$ \\
\hline A. Avec les membres de sa famille, Paul se montre désagréable. \\
montrer désai, c'ést dans sa nature de se & & \\
B- Ce sont les membres de sa famille qui & & \\
induisent qu'il se montre désagréable. & & \\
C- Ce sont des círconstances particulières & & \\
qui l'amènent àre montrer désagréable. & & \\
\hline
\end{tabular}




\begin{tabular}{|c|c|c|}
\hline & $\begin{array}{c}\text { Bon } \\
\text { candidat }\end{array}$ & $\begin{array}{l}\text { Mruvais } \\
\text { candidat }\end{array}$ \\
\hline \multicolumn{3}{|l|}{ 9. Avec ses amis, Paul se montre etroit d'esprit. } \\
\hline \multicolumn{3}{|l|}{$\begin{array}{l}\text { A- C'est en lui, s'est dans sa nature de se } \\
\text { montrer étroit d'esprit. }\end{array}$} \\
\hline \multicolumn{3}{|l|}{$\begin{array}{l}\text { B- Ce sont ses amis qui induisent qu'il se } \\
\text { montre ftroit d'esprit. }\end{array}$} \\
\hline $\begin{array}{l}\text { C-Ce sont des circonstances particulières } \\
\text { qui l'amènent à se montrer étroit d'esprit. }\end{array}$ & & \\
\hline
\end{tabular}

\begin{tabular}{|c|c|c|}
\hline & $\begin{array}{c}\text { Bon } \\
\text { candidat }\end{array}$ & $\begin{array}{l}\text { Mauvais } \\
\text { candidat }\end{array}$ \\
\hline \multicolumn{3}{|c|}{ 10. Face dे des inconnus; Panl se montre sâr de lut. } \\
\hline $\begin{array}{l}\text { A-C'est en lui, c'est dans sa nature de se } \\
\text { montrer strr de lui. }\end{array}$ & & \\
\hline $\begin{array}{l}\text { B- Ce sont les inconnus qui induisent qu'il se } \\
\text { montre sâr de lui. }\end{array}$ & & \\
\hline $\begin{array}{l}\text { C-Ce sont des circonstances particulières } \\
\text { qui l'aménent à se montrer sûr de lui. }\end{array}$ & & \\
\hline
\end{tabular}

